\title{
The Examination of Slo-pitch Hitting Movement Coordination
}

\author{
Tom Wu ${ }^{1 *}$, Pierre Gervais ${ }^{2}$, Pierre Baudin ${ }^{2}$ and Marcel Bouffard ${ }^{2}$ \\ ${ }^{1}$ Department of Movement Arts, Bridgewater State University, USA \\ ${ }^{2}$ Faculty of Physical Education and Recreation, University of Alberta, Canada
}

*Corresponding author: Tong-Ching Tom Wu, Ph.D, Associate Professor, Department of Movement Arts, Health Promotion and Leisure Studies, Adrian Tinsley Center, Bridgewater State University, Bridgewater, MA 02325, USA

Submission: 海 December 04, 2017; Published: 䤈December 14, 2017

\begin{abstract}
This study developed an interdisciplinary approach by utilizing the principles of ecological task analysis and movement coordination from areas of motor leaning and biomechanics to examine the skill of slo-pitch softball hitting. The purpose of this study was to examine the influence of a task constraint, stride technique, and an environmental constraint, pitched ball location, on the participants' movement patterns. Ten elite male softball players participated in the study and a two-way ANOVA of 2 locations of pitch (inside and outside) x 3 strides (open, parallel and closed) repeated measure study was conducted. The results showed that participants demonstrated different joint movements and different coordination patterns in slo-pitch hitting. Hence, this study supports the rationale of ecological task analysis. Further, a Euclidean distance analysis was conducted to evaluate the degree of dissimilarity between the individual and group mean results in attempt to better understand the generalizability. The results indicated that participants generally showed a low degree of dissimilarity; hence, coaches and educators may apply the findings to other players. A similar interdisciplinary approach is warranted for future research studies on other sport skills or health conditions in order to better understand the mechanics of human motion.
\end{abstract}

Keywords: Constraint; Coordination; Hitting; Movement; Softball

\section{Introduction}

In the area of motor learning, Newell [1] proposed three categories of constraints (organismic, environmental and task) that interact closely with each other and have an influence on the development of coordination and control. Davis \& Burton [2] developed ecological task analysis based on the approach taken by Newell [1]. Ecological task analysis (ETA) uses a dynamic system approach to examine the stability and change of a performer's movement form as a result of dynamic interactions between the performer, environment and task [2,3]. In sport settings, the performer constraint is the characteristics of the performer (player) such as strength and skill levels [2-4]. Environmental constraint is the environmental condition in which the performer performs the task. This includes the performer's surrounding environmental conditions such as the field of play, sun, wind, and temperature and also those related to the opposing team such as location of the pitched ball to the batter and defensive player's positions on the field. Task constraint is establishing and identifying specific task goals for the performer such as hitting a ball with an open stride technique.

In ecological task analysis, the performer's movement patterns are based on the results of the dynamic interactions between the performer, environment and task constraints. Therefore, if one of the constraints is changed, the performer's performance outcome will be changed as well [2-4]. For example, if a pitcher threw an inside pitch to a batter, the batter could use an open stride to hit the ball. However, if the pitcher threw an outside pitch to the batter instead, the batter could then change his stride to the closed stride and still be able to accomplish the same task goal of hitting the ball. Hence, there is more than one movement solution to accomplish the task [2-4]. This type of analysis provides an intrinsic motivation to the performer and uses a performer-oriented approach [4]. Conversely, in traditional task analysis, the instructor describes and identifies the components of the task, and the performer is instructed to accomplish the task in a sequence of specific steps and movements [2]. The instructor directs the performer to accomplish the task with only one best possible biomechanical movement pattern, so the traditional task analysis uses a teacher-oriented approach [3].

In the area of sports biomechanics, Northrip et al. [5] defined coordination as a proper sequence of force production to produce an optimal outcome to achieve a task goal, and the examination of timing and sequencing of the movement can provide a fundamental understanding in coordination. Hudson [6] developed a shared 
positive contribution (SPC) technique to assess coordination by examining the timing and sequencing of a movement. The shared positive contribution shows the degree to which two segments operate either simultaneously or sequentially. A SPC of $0 \%$ indicates a sequential type of movement coordination pattern, and a SPC of $100 \%$ indicates a simultaneous type of movement coordination pattern. A sequential type of movement occurs when a proximal segment reaches its peak velocity before a distal segment initiates its movement. This type of movement occurs when the focus of the task is on velocity, a light object is used, or when it is an open kinetic chain movement. The open kinetic chain movement is defined by the end segment of a sport skill movement that can move freely in space. A simultaneous type of movement occurs when all segments initiate the movements at the same time. This type of movement occurs when the focus of the task is on accuracy, a heavy object is used, or when it is a closed kinetic chain movement. The closed kinetic chain movement in a sport skill is defined by an end segment movement that experiences a resistive force. Hence, the free motion of the end segment in space is restricted or constrained [7-9]. A sport skill movement sometimes cannot be classified as entirely sequential (SEQ) or entirely simultaneous (SIM). The skill movement may be a combination of both types, so it falls in a continuum ranging from the sequential to simultaneous [6,7]. Hudson [6] and Malone et al. [10] adapted shared positive contribution (SPC) technique in an attempt to classify the body segmental movement pattern objectively, so comparison can be made between different sports skills, which enable coaches and teachers to provide better coaching and teaching instructions. Therefore, the purpose of this study was to examine the slo-pitch hitting movement coordination using the shared positive contribution technique to evaluate players' movement coordination under different task (stride technique) and environmental constraints (location of pitch). Further, each participant's results were compared to the mean to evaluate the degree of dissimilarity between the individuals and the group using a Euclidean distance analysis [11]. These results enabled assessment of whether the group means performance results could be used to generalize to all participants.

\section{Methods}

\section{Participants and experimental design}

Ten right-handed skilled male slo-pitch players (mean age $=33.7 \pm 5.0$ years; height $=1.8 \pm 0.1 \mathrm{~m}$; weight $=93.5 \pm 21.0 \mathrm{~kg}$; and playing experience $=12.7 \pm 5.5$ years) who played in competitive A/B division volunteered to participate in the study. This research study was approved by the research ethics institutional review board, and written informed consent was obtained from each participant prior to their participation in the study. All participants were healthy and excluded from the study if they were currently injured or had a history of chronic injuries related to their training. Twelve joint reflective markers were placed on various body joints which include right and left acromio-clavicular joints, right and left medial epicondyles of the humerus, right and left lateral epicondyles of the humerus, right and left anterior superior iliac spines, right and left medial epicondyles of the tibia, right and left lateral epicondyles of the tibia, and right and left malleoli. Additionally, two reflective markers were placed on an Easton Cyclone SK37 0.78kg and $0.86 \mathrm{~m}$ (28 oz and 34") bat at the top and bottom of the bat, respectively.

\section{Apparatus and experiments}

This study took place in an indoor field house to control the influence of air forces. Wu \& Gervais [12,13] reported that a slopitch pitcher's stride length was approximately equal to $0.8 \mathrm{~m}$, so a Jugs Lite-Flite pitching machine (Jugs Softball, Jug Inc., Tualatin, OR) was placed $14.4 \mathrm{~m}$ away from the participant to account for the difference of the pitching distance to the batter. Twenty-four Jugs Lite-Flite indoor softballs, $0.30 \mathrm{~m}(12$ "), were used and small strips of reflective tape were placed on the surface of the balls (weight of the ball with the tape $=0.07 \mathrm{~kg}$ ) to enable identifying the instant of ball contact. Each ball was thrown by the pitching machine at a speed of approximately $13.5 \pm 0.7 \mathrm{~m} / \mathrm{s}$ with an arc trajectory of $2.7 \pm 0.2 \mathrm{~m}$ to two different strike zone locations (inside or outside). A blue mat was placed in front of the pitching machine so that the batter could not anticipate ball's pitch location. Participants performed their regular warm-up routine and took batting practice until they were ready for testing. Each participant stood at their own comfortable location in the batter's box with their own natural stance. Participants were instructed to use either a closed, open or parallel stride technique to hit the ball. The closed stride is defined as when the batter strides toward the home plate at an angle greater than $30^{\circ}$ in a clockwise direction. The open stride is defined as when the batter strides away from the home plate at an angle greater than $30^{\circ}$ in a counter-clockwise direction. The parallel stride is defined as when the batter strides toward the pitching machine at an angle of less than $30^{\circ}$ in either a clockwise direction or a counter-clockwise direction, Figure 1.

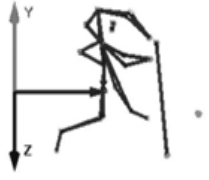

A

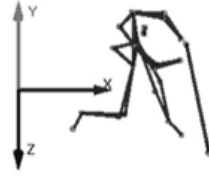

B

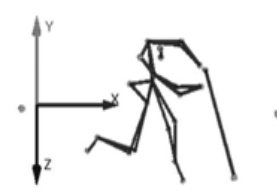

c
Figure 1: Three different stride techniques: A) open, B) parallel and C) closed.

The stride angles from the Qualisys data were used to examine whether the participants had performed the requested stride technique. During the testing, each participant hit six balls in each of six conditions to ensure reliability of each participant's performance [14]. The participant had 30 seconds to rest between each ball, and one minute to rest between each condition. The influence of fatigue and the risk of injury were minimal in this study. Since there were a total of two different strike zone locations (inside or outside) and three different stride techniques (open, parallel or closed), a total of 36 balls were hit by each participant. Hence, a total of 360 trials were collected from 10 participants in this study. The order of the strike zone locations and stride technique were randomized to reduce any order effect. 
A three-dimensional (3D) analysis was conducted with an 8-camera Qualisys motion capture system (Pro Reflex MCU 240, Qualisys AB, Sweden) that was operated at $240 \mathrm{~Hz}$. Six cameras were placed approximately $60^{\circ}$ apart around the participant and two cameras were placed on top of a concourse to provide an overhead view of the batting motion. The size of calibration volume was approximately $2.5 \mathrm{~m}$ (X-direction) x $2.5 \mathrm{~m}$ (Y-direction) x $2.5 \mathrm{~m}$ (Z-direction), and a wand calibration technique was used to calibrate the volume. The calculations of the 3D coordinates for each body segment rotational angles were presented by Wu et al. [11]. The data were smoothed with $4^{\text {th }}$ order Butterworth filter, and the optimal cut-off frequency was determined for each coordinate using residual analysis [15]. The cut-off frequency for the $\mathrm{x}$-coordinate ranged from 6.3 to $12.2 \mathrm{~Hz}$; the y-coordinate ranged from 6.1 to $11.6 \mathrm{~Hz}$, and the z-coordinate ranged from 6.3 to $10.8 \mathrm{~Hz}$.

For determining type of movement pattern, a shared positive contribution (SPC) or a reversed shared positive contribution (RSPC) was calculated for each trial for each pair of joint rotational angles (lower body and trunk, and trunk and upper body). The shared positive contribution is based on the rationale that the proximal segment or joint initiates its movement before the distal segment or joint. However, this principle does not apply to all striking and throwing sport skills [16-19]. Hence, this study has included the reversed shared positive contribution (RSPC), in which the distal segment or joint initiates its movement before the proximal segment or joint. Both shared positive contribution and reversed shared positive contribution show the degree to which two segments or joints move either simultaneously or sequentially. A SPC or a RSPC of $0 \%$ indicates a sequential type of movement coordination pattern, and a SPC or a RSPC of $100 \%$ indicates a simultaneous type of movement coordination pattern. The two body segments that are used for the assessment of the movement coordination pattern must first be identified as either the proximal segment or joint or the distal segment or joint. Welch, et al. [20] examined baseball hitting mechanics and indicated that baseball hitting was a closed kinetic chain movement starting when the stride foot was planted on the ground. The sequence of segmental or joint movements to execute a hitting skill starts with the stride foot contact followed by the hip rotation then the shoulder rotation (trunk rotation) concluding with the arm rotation [20-24]. Since the hitting skill is a closed kinetic chain movement, the sequence of body movement is from the lower body segments/joints to the upper body segment/joints. Hence, in this study the proximal joint was defined as the joint that was closer to the fixed point (i.e. ground) of the kinetic link system and the distal joint was defined as the joint that was furthest away from the fixed point of the kinetic link system. The shared positive contribution was defined as a proximal to distal pattern where movement was initiated by the proximal joint followed by the distal, and the reversed shared positive contribution was defined as a distal to proximal pattern where movement was initiated by the distal joint followed by the proximal. Both shared positive contribution and reversed shared positive contribution were determined as the time when both proximal and distal joints were in a propulsion phase divided by the time when either joints was in the propulsion phase. The propulsion phase was the time interval defined from the zero velocity or zero acceleration, if the zero velocity was not present and sped up to maximum absolute joint velocity prior to ball contact $[6,10]$. The selections of the time scale points were determined in a reversed direction from the ball contact to zero velocity or zero acceleration if zero was not present. The instant of ball contact was located first and then the instant of maximum absolute joint velocity with its corresponding zero velocity or zero acceleration, if zero velocity was not present, were determined accordingly, Figure 2.

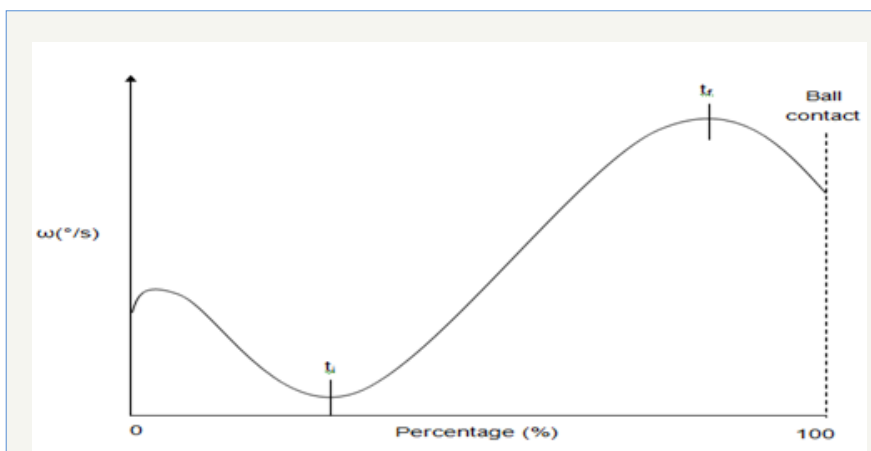

Figure 2: Normalized for time on the abscissa $(0 \%$ is the start of front foot striding toward the ball and $100 \%$ is at ball contact). The propulsion phase was defined from $t_{i}$ that corresponds to zero velocity or zero acceleration, if the zero velocity was not present, with increasing speed to $t_{f}$ that corresponds to the maximum absolute angular velocity prior to ball contact. The selections of the time points $\left(t_{i}\right.$ and $\left.t_{f}\right)$ were determined in a reversed direction from the ball contact to zero velocity or zero acceleration if zero was not present. The instant of ball contact was located first and then the instant of maximum absolute joint velocity with its corresponding zero velocity or zero acceleration, if zero velocity was not present were determined accordingly. The propulsion phase $\left(t_{p}\right)$ was equal $t_{f}-t_{i}$ with both $t_{i}$ and $t_{f}$ correspond to the original non-normalized time scale points (i.e. seconds).

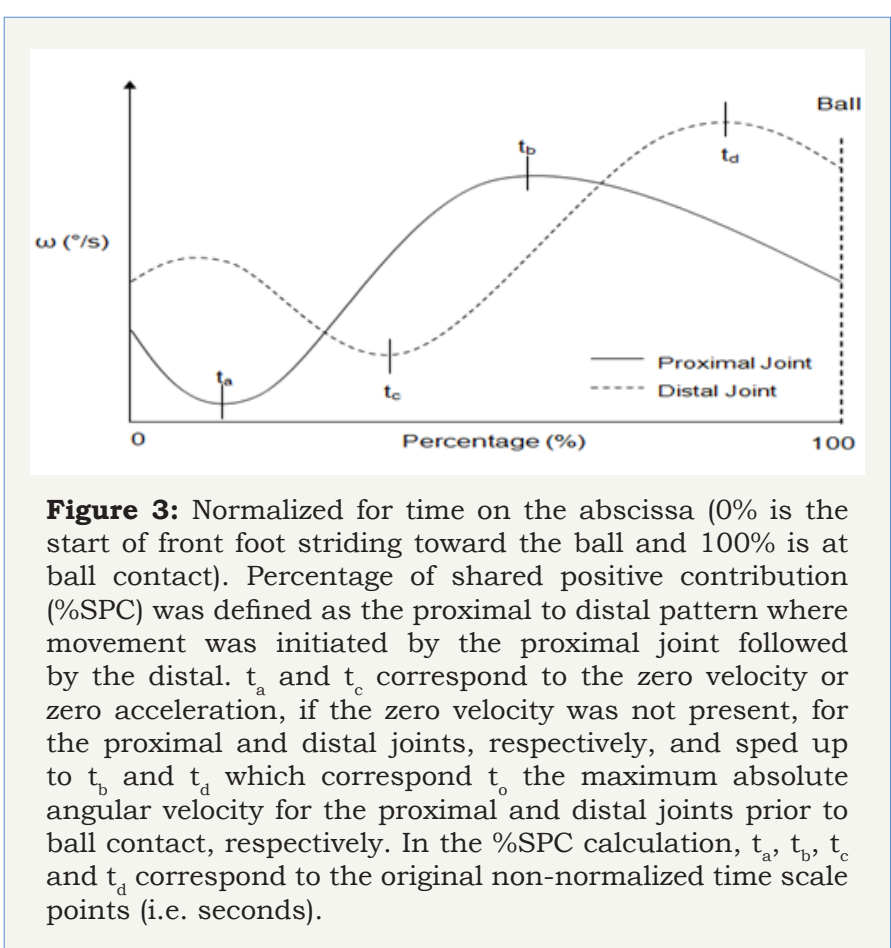


The shared positive contribution (SPC) and the reversed shared positive contribution (RSPC) were calculated as the following (see Figure 3 \& Figure 4 for an illustration of the SPC and RSPC, respectively).

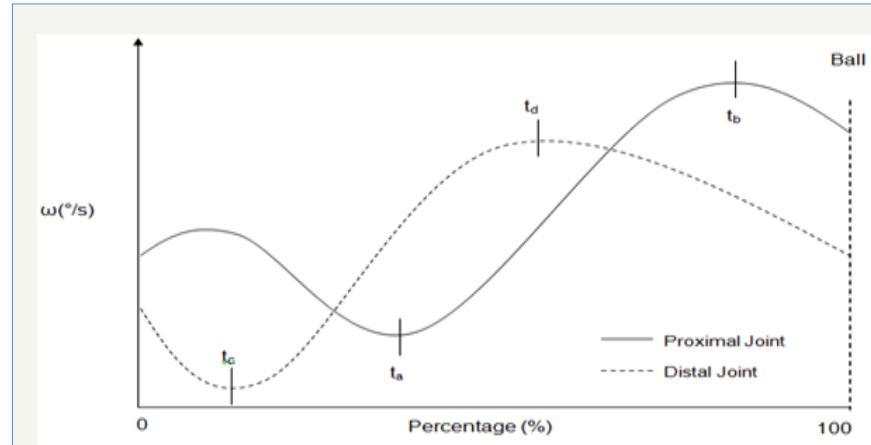

Figure 4: Normalized for time on the abscissa $10 \%$ is the start of front foot striding toward the ball and $100 \%$ is at ball contact). Percentage of reversed share positive contribution (\%RSPC) was defined as the distal to proximal pattern where movement was initiated by the distal joint followed by the proximal. $t_{a}$ and $t_{c}$ correspond to the zero velocity or zero acceleration, if the zero velocity was not present, for the proximal and distal joints, respectively, and sped up to $t_{b}$ and $t_{d}$ which correspond to the maximum absolute angular velocity for the proximal and distal joints prior to ball contact, respectively. In the \%RSPC calculation, $t_{a}, t_{b}, t_{c}$ and $t_{d}$ correspond to the original non-normalized time scale points (i.e. seconds).

$$
\begin{aligned}
& \% \mathrm{SPC}=\left[\left(\mathrm{t}_{\mathrm{b}}-\mathrm{t}_{\mathrm{c}}\right) /\left(\mathrm{t}_{\mathrm{d}}-\mathrm{t}_{\mathrm{a}}\right)\right] \times 100 \\
& \% \mathrm{RSPC}=\left[\left(\mathrm{t}_{\mathrm{d}}-\mathrm{t}_{\mathrm{a}}\right) /\left(\mathrm{t}_{\mathrm{b}}-\mathrm{t}_{\mathrm{c}}\right)\right] \times 100
\end{aligned}
$$

Where in both \% SPC and \% RSPC calculations, $t_{a^{\prime}} t_{b}, t_{c}$ and $t_{d}$ correspond to the original non-normalized time scale points (i.e. seconds). The $t_{a}$ and $t_{c}$ correspond to the zero velocity or zero acceleration, if zero velocity was not present, for the proximal and distal joints, respectively. The $t_{b}$ and $t_{d}$ correspond to the maximum absolute joint angular velocity for the proximal and distal joints, respectively. Based on a sampling rate of $240 \mathrm{~Hz}$ and using the normalized time on the abscissa, it is estimated that the consequences of a one frame error in locating t would correspond to 0.0042 seconds in the non-normalized time scale or an error equal to $0.42 \%$ (Figure $3 \& 4$ ).

\section{Statistical Analysis}

A two-way repeated measure ANOVA (2 locations of pitch $\mathrm{x} 3$ strides) was conducted at $\alpha=0.05$ on combined percentage value for both SPC and RSPC for movement pattern coordination at ball contact for lower body and trunk segments and trunk and upper body segments. If a significant difference was found in the ANOVA test, pair wise comparisons were conducted using a t-test with the Bonferroni adjustment at $\alpha=0.05 / c$, where $c$ was the number of contrasts [25]. In each of six conditions, a rescaled Euclidean distance analysis was conducted on combined percentage value for both SPC and RSPC for movement pattern coordination at ball contact for lower body and trunk segments and trunk and upper body segments between each participant and group mean performance. The degree of dissimilarity enabled us to assess if the group mean performance could be generalized to all participants. The rescaled Euclidean distance analysis was the measure of distance between dependent variables for individual performance versus group mean performance, and it ranged between values of 0 for no dissimilarity to a value of 1 for maximum dissimilarity [11].

\section{Results}

Table 1: Combined \% SPC and \% RSPC of movement pattern coordination for different locations of pitch.

\begin{tabular}{|c|c|c|c|}
\hline $\begin{array}{c}\text { Movement Pattern } \\
\text { Coordination }\end{array}$ & $\begin{array}{c}\text { Inside } \\
\text { Pitch }\end{array}$ & Outside Pitch & $\mathbf{p}$ \\
\hline Lower body and trunk (\%) & $26.4 \pm 41.0$ & $28.8 \pm 33.9$ & 0.85 \\
\hline Trunk and upper body (\%) & $32.2 \pm 29.4$ & $33.4 \pm 29.4$ & 0.85 \\
\hline
\end{tabular}

*Statistical significant at $\mathrm{p}<0.05$

The results of two-way repeated measured ANOVA (2 locations of pitch $\times 3$ strides) statistical analysis revealed that there was no statistically significant difference in combined percentages of SPC and RSPC between the lower body and trunk segments for the location of pitch. Similarly no statistically significant differences were observed between the trunk and upper body segments, Table 1. Further, the results showed that there was also no significant difference for each pair of body segment between the three stride techniques. The combined percentages of SPC and RSPC of the lower body and trunk segments for the open, parallel and close strides were $22.6 \pm 39.9 \%, 21.1 \pm 27.1 \%$, and $39.1 \pm 42.3 \%$, respectively. Also, the combined percentages of SPC and RSPC of the trunk and upper body segments for the open, parallel and close strides were $23.3 \pm 25.7 \%, 44.9 \pm 31.1 \%$, and $30.0 \pm 27.3 \%$, respectively. Additionally, no statistically significant differences were found in the two-way interaction effect between the location of the pitch and stride for both pairs of body segments.

A Euclidean distance analysis was conducted on combined percentages of SPC and RSPC for both lower body and trunk, and trunk and upper body joints in each of six conditions. The results indicated that across six different hitting conditions $30.0 \%$ of participants showed a degree of dissimilarity between 0 and 0.20 , and $53.0 \%$ of participants showed a degree of dissimilarity between 0.21 and 0.40 . Further, $12.0 \%$ of participants showed a degree of dissimilarity between 0.41 and 0.60 , and $5.0 \%$ of participants showed a degree of dissimilarity between 0.61 and 0.80 . Cumulatively, approximately $83.0 \%$ of participants illustrated a degree of dissimilarity below 0.40 , and $95.0 \%$ of participants illustrated a degree of dissimilarity below 0.60. Therefore, participants generally showed that their individual combined percentages of SPC and RSPC results were similar to the group mean combined percentages of SPC and RSPC results.

\section{Discussion and Implications}

The purpose of this study was to examine the slo-pitch hitting movement coordination using the shared positive contribution technique to evaluate players' movement coordination under 
different task (stride technique) and environmental constraints (location of pitch). The results have indicated that there were no statistically significant differences between different locations of pitch and stride techniques for two pairs of body segment: lower body and trunk, and trunk and upper body. Typical sport biomechanics research studies use a traditional task analysis approach which suggests that there is only one best possible movement coordination to perform a sport skill [3]. Conversely, this study was guided by the concept of ecological task analysis from the motor learning area. Ecological task analysis uses a dynamic system approach to examine the stability and change of the performer's movement form as a result of dynamic interactions between the three major constraints of task, performer and environment $[2,3]$. In this study the task constraint was the stride technique, and the environmental constraint was the pitched ball location. These constraints were evaluated to examine their influence on the movement coordination of slo-pitch soft ball hitting. Participants showed that they were able to accomplish their task goals using either sequential or simultaneous coordination, Figure 5, and it was further observed that participants used both types of movement patterns, either proximal to distal pattern (SPC) or distal to proximal (RSPC), Figure 6.

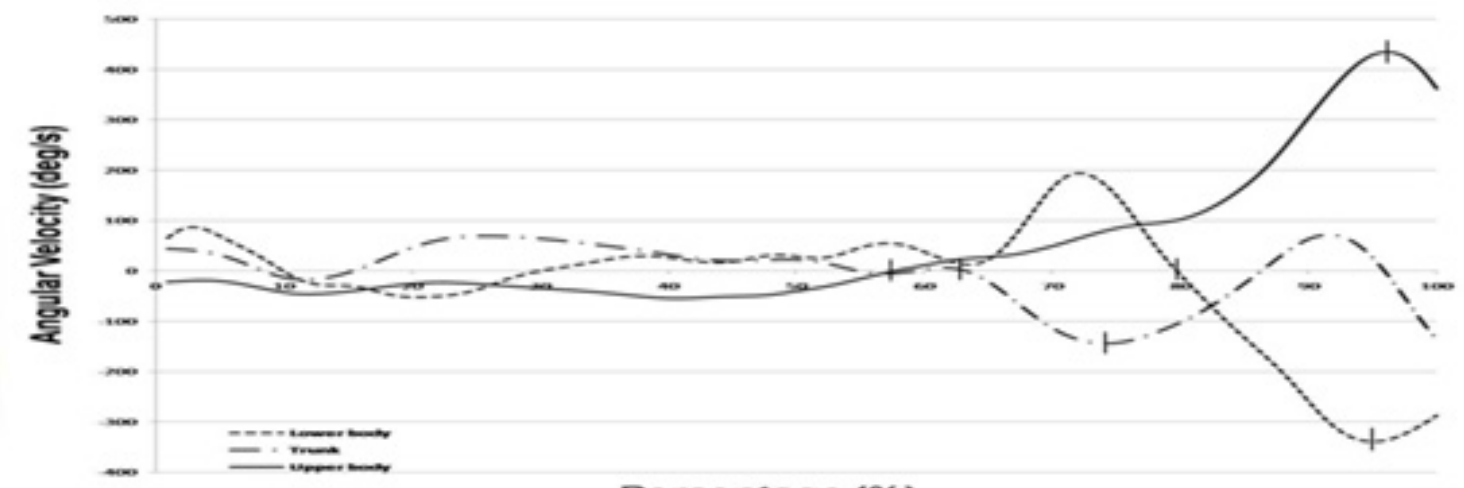

Figure 5: Normalized for time on the abscissa $(0 \%$ is the start of front foot striding toward the ball and $100 \%$ is at ball contact). The graph is an example of movement coordination pattern for a participant hitting an outside pitch using parallel stride. The lower body and trunk joints show a RSPC of $-21 \%$ in a sequential movement coordination pattern while the trunk and upper body joints show a RSPC of $222 \%$ in a simultaneous movement coordination pattern. The vertical lines indicate the beginning and end of the propulsion phase prior to ball contact.

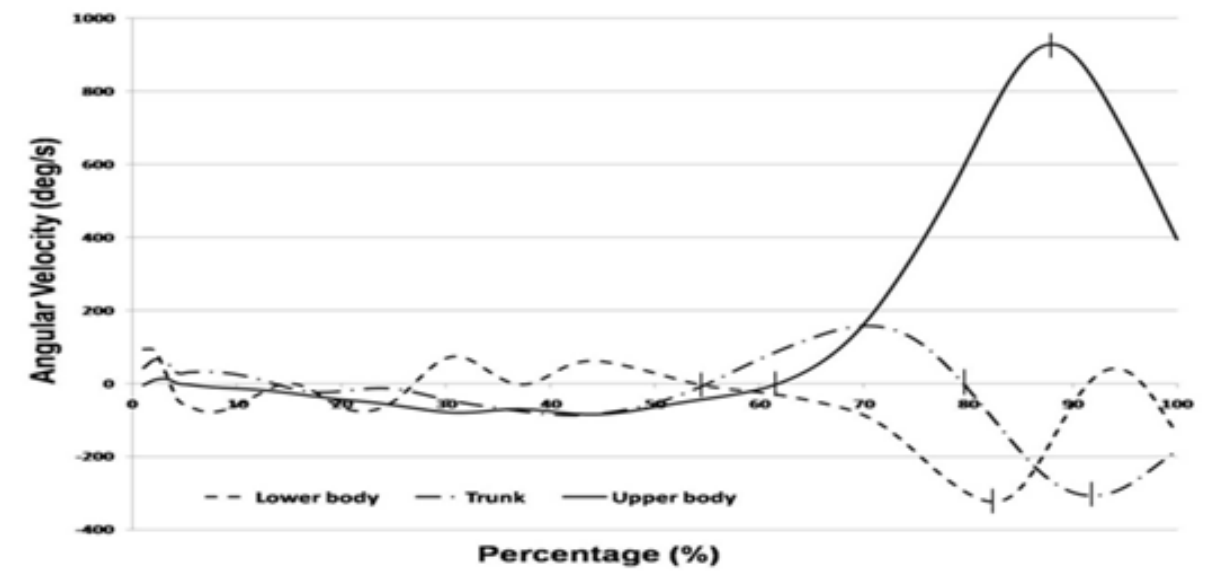

Figure 6: Normalized for time on the abscissa ( $0 \%$ is the start of front foot striding toward the ball and $100 \%$ is at ball contact.). The graph is an example of movement coordination pattern for a participanthitting an inside pitch using open stride. The lower body and trunk joints show a SPC of $8 \%$ in a sequential movement coordination pattern while the trunk and upper body joints show a RSPC of $30 \%$ in a sequential movement coordination pattern. The vertical lines indicate the beginning and end of the propulsion phase prior to ball contact.

Coordination is one of the key concepts in the study of motor learning and development. Bernstein [26], a Russian physiologist, defined coordination as "the organization of control of the motor apparatus" (p. 127) and proposed the development of coordination as "the process of mastering redundant degrees of freedom of the moving organ, in other words, its conversion to a controllable 
system" (p. 127). Bernstein (1967) viewed the development of coordination as a result of a learner being able to utilize various methods "in order to reduce the number of degrees of freedom at the periphery to a minimum" (pp. 107-108), and the degrees of freedom was defined as the number of mechanical movements that the learner can use to achieve the task objective [27]. Then the learner gradually releases all restrictions on the degrees of freedom of the movement control. This process is known as shifting from "freezing" to "freeing" in the number of degrees of freedom in movement control. Finally the learner utilizes and exploits this movement control to execute the task successfully [27]. However, Broderick \& Newell [28] found that the concepts on coordination as proposed by Bernstein [26] were not entirely true. Broderick \& Newell [28] conducted a study to examine the learning effect of beginner's coordination in the skill of basketball bouncing. Participants showed an increase in the number of degrees of freedom which resulted in a change in their coordination pattern, and this change of coordination pattern was mainly due to influence of the task constraint factor.

This present study was guided by Hudson [6] shared positive contribution (SPC) technique to assess coordination patterns by examining the temporal sequencing of the movement. From the results of this study the participants demonstrated two types of body joint movement. Some participants showed a proximal to distal type of joint movement either with a sequential or a simultaneous coordination pattern while other participants showed a distal to proximal type of joint movement also either with a sequential or a simultaneous coordination pattern. Therefore, participants showed both types of joint movements and both types of movement coordination pattern across six different conditions. The results showed that participants may use multiple movement coordination patterns to achieve the task goal successfully. Since all participants were skilled players and did not have any practice in each condition before the testing, the individual participant's change in coordination pattern across six different conditions was the result of their skill adaptation due to the influence of both task and environmental constraints. Therefore, this study supports Broderick \& Newell [28] rationale that the participant's change in coordination pattern is influenced by various constraints in action and participants can use multiple movement solutions to achieve the same task goal, which coincides with the principles of ecological task analysis. Specifically, participants can use either a sequential or a simultaneous type of coordination pattern, and these types of coordination pattern can be performed with a proximal to distal type of joint sequencing or a distal to proximal type of joint sequencing. Further, participants can use open, parallel or close stride technique to hit the ball with different body movement coordination, so this study encourages participants to explore their own movement solutions to achieve their task goal.

\section{Conclusion}

This study used 10 elite slo-pitch batters to examine the influence of task (stride technique) and environmental (pitched ball location) constraints on movement kinematics. The results showed that the pitched ball location and stride techniques did not have an influence on the movement kinematics. Therefore, the results of this study recommend players explore different stride techniques, and the selected stride technique may simply be based on what they are most familiar with to hit the ball. Regardless the type of selected stride technique, this study suggested that players should explore their own movement coordination pattern that enables them to hit the ball.

In terms of generalizability of the study, the majority of participants showed a degree of dissimilarity that is less than or equal to 0.40 when comparing their individual result to the group mean result. This study indicated that the participants from this study were quite similar to each other and homogeneous as a group. Therefore, participants generally showed a strong similar response to the treatment and as such, base on the knowledge about the degree of generalizability coaches and researchers may utilize the findings from this study to other players. Since players can use different types of stride technique to hit the ball, future research should examine players' movement coordination in other sport skills and understand how players can perform those skills successfully.

\section{Acknowledgement}

The authors would like to thank Edmonton and Big League Apparel \& Sports slo-pitch players for participating in the study. In addition, the authors acknowledge equipment support from the Softball-tips.com \& Baseballtips.com and the research development support from the Human Performance Scholarship Fund, University of Alberta.

\section{References}

1. Newell KM (1986) Constraints on the development of coordination. In: Wade MG, Whiting HTA (Eds.), Motor development of children; Aspects of coordination and control, Martin Nijhoff, Amsterdam, USA, pp. 341361.

2. Davis WE, Burton AW (1991) Ecological task analysis: Translating movement behaviour theory into practice. Adapted Physical Activity Quarterly 8(2): 154-177.

3. Balan CM, Davis WE (1993) Ecological task analysis-An approach to teaching physical education. Journal of Physical Education, Recreation \& Dance 64(9): 54-61.

4. Burton AW, Davis WE (1996) Ecological task analysis utilizing intrinsic measures in research and practice. Human Movement Science 15(2): 285-314.

5. Northrip JW, Logan GA, McKinney WC (1983) Analysis of sport motion: anatomic and biomechanics perspectives ( $3^{\text {rd }}$ edn), Brown, Dubuque, USA.

6. Hudson JL (1986) Coordination of segments in the vertical jump. Med Sci Sports Exerc 18(2): 242-251.

7. Kreighbaum E, Barthles K (1996) Biomechanics, a qualitative approach for studying human movement ( $4^{\text {th }}$ edn), Burgess Publishing Company, Minneapolis, USA.

8. Luttgens K, Wells KF (1982) Kinesiology: scientific basis of human motion ( $7^{\text {th }}$ edn), Saunders, Philadelphia, USA.

9. Morehouse LE, Cooper JM (1950) Kinesiology. St Louis, Mosby, USA. 
10. Malone LA, Gervais PL, Steadward RD (2002) Patterns of segment coordination in wheelchair basketball shooting. Journal of Human Movement Studies 43: 211-227.

11. Wu T, Gervais P, Baudin P, Bouffard M (2011) The effects of stride technique and pitch location on slo-pitch batting. Sports Biomech 10(14): 351-360.

12. Wu T, Gervais P (2006) The effects of spin on ball trajectories in slopitch. XIV ${ }^{\text {th }}$ Biennial Conference for the Canadian Society of Biomechanics. Canadian Society of Biomechanics, Waterloo, p. 125.

13. Wu T, Gervais P (2008) An examination of slo-pitch pitching trajectories. Sports Biomechanics 7(1): 88-99.

14. Hopkins WG (2000) Measures of reliability in sports medicine and science. Sports Med 30(1): 1-15.

15. Wells RP, Winter DA (1980) Assessment of signal and noise in the kinematics of normal, pathological, and sporting gaits. In: Human locomotion, Pathological gait to the elite athlete, Canadian Society of Biomechanics, London, pp. 92-93.

16. Alexander MJ, Haddow JB (1982) A kinematic analysis of an upper extremity ballistic skill: the windmill pitch. Can J Appl Sport Sci 7(3): 209217.

17. Marshall RN, Elliott BC (2000) Long-axis rotation: the missing link in proximal-to-distal segmental sequencing. J Sports Sci 18(4): 247-254.

18. Milburn PD (1982) Summation of segmental velocities in the golf swing. Med Sci Sports Exerc 14(1): 60-64.
19. Van Gheluwe B, de Ruysscher I, Craenhals J (1987) Pronation and endorotation of the racket arm in a tennis serve. In: (B. Jonsson Eds.), Biomechanics X-B, Champaign: Human Kinetics, pp. 667-672.

20. Welch CM, Banks SA, Cook FF, Draovitch P (1995) Hitting a baseball: a biomechanical description. J Orthop Sports Phys Ther 22(5): 193-201.

21. Bennett G, Yeager C (2000) The stride is key to hitting. Strategies 13(4): 5-7.

22. Hay JG (1978) The biomechanics of sports techniques. Prentice-Hall, USA.

23. Pardee $P$ (1980) Techniques for hitting in fast pitch softball. Women's Coaching Clinic 3(7): 16-19.

24. Shapiro R (1974) Cinematographic analysis of batters hitting base balls thrown at different heights and velocities. The Pennsylvania State University, University Park, USA.

25. Maxwell SE, Delaney HD (1990) Designing experiments and analyzing data: a model comparison perspective. Wadsworth Publishing Company, Belmont, USA

26. Bernstein N (1967) The coordination and regulation of movement.. New York, USA.

27. Newell KM, Vaillancourt DE (2001) Dimensional change in motor learning. Hum Mov Sci 20(5): 695-715.

28. Broderick MP, Newell KM (1999) Coordination patterns in ball bouncing as a function of skill. J Mot Behav 31(2): 165-189. 